\title{
Prenatal Diagnosis and Neonatal Monitoring of a Fetus with Glutaric Aciduria Type II Due to Electron Transfer Flavoprotein $(\beta$-Subunit) Deficiency
}

\author{
SEIJI YAMAGUCHI, NOBUO SHIMIZU, TADAO ORII, TOSHIYUKI FUKAO, \\ YASUYUKI SUZUKI, KEIKO MAEDA, TAKASHI HASHIMOTO, STEPHEN F. PREVIS, AND \\ PIERO RINALDO \\ Department of Pediatric's, Gifu University School of Medicine, Gifu 500, Japan [S.Y., N.S., T.O., T.F., Y.S.]; \\ Division of Pediatrics, Shizuoka-Saiseikai General Hospital, Shizoka 420, Japan [K.M.]; Department of \\ Biochemistrv. Shinshu University School of Medicine, Matsumoto, Nagano 390, Japan [T.H.]; and Department \\ of Genetics, Yale University School of Medicine, New Haven, Connecticut 06510 [S.F.P., P.R.]
}

\begin{abstract}
The prenatal diagnosis of a male fetus with glutaric aciduria type II and the time course of metabolite urinary excretion, starting immediately after birth, are described. Prenatal diagnosis was undertaken at the 17 th wk of gestation by immunoblot analysis and pulse labeling experiments of amniocytes and, retrospectively, by stable isotope dilution analysis of six metabolites in amniotic fluid. The results were as follows: 1 ) The immunochemical analysis on cultured amniocytes showed that the fetus, as the previous index case in this family, was affected with a deficiency of the $\beta$-subunit of electron transfer flavoprotein. 2) Glutarate concentration was significantly increased in the cell-free supernatant of the amniotic fluid. In the postnatal period, most of the organic acids and acylglycines characteristic of the disorder appeared in urine within a week, although an increased excretion of hexanoylglycine was the only biochemical abnormality detectable in the first urine sample collected at $9 \mathrm{~h}$ after birth. Growth and development of this infant were normal during the following 6 mo of life, when he was receiving oral supplementation with L-carnitine and riboflavin. It should be underscored that transient abnormalities in routine blood tests (glutamic oxaloacetic transaminase, lactate dehydrogenase, and creatine phosphokinase) were present soon after birth, despite his asymptomatic clinical course. Early detection and aggressive treatment could be effective in such a form of glutaric aciduria type II. (Pediatr Res 30: 439-443, 1991)
\end{abstract}

Abbreviations

GAII, glutaric aciduria type II

ETF, electron transfer flavoprotein

$\alpha$-ETF, $\alpha$-subunit of ETF

$\beta$-ETF, $\beta$-subunit of ETF

GC/MS, gas chromatography mass spectrometry

GOT, glutamic oxaloacetic transaminase

LDH, lactate dehydrogenase

Received March 8, 1991; accepted July 3, 1991.

Correspondence: Seiji Yamaguchi, M.D. Department of Pediatrics, Gifu University School of Medicine, 40 Tsukasa-machi, Gifu 500, Japan.

Supported by grants for the promotion of science research (01570522) from the Ministry of Education, Science and Culture of Japan and for studies on inherited disorders (630104) from the Ministry of Health and Welfare, Japan, and by a grant (2-11-19) from National Center of Neurology and Psychiatry (NCNP) of the Ministry of Health and Welfare, Japan. S.F.P. and P.R. were supported in part by a grant from the Joseph Drown Foundation and the BRSG Grant RR 05358 awarded by the Biomedical Research Support Grant Program, Division of Research Resources, National Institutes of Health.
CPK, creatine phosphokinase

GAII is an inherited disorder of organic acid metabolism that is caused by disturbances in several acyl-CoA dehydrogenation steps, such as medium-, short-, and long-chain acyl-CoA dehydrogenases, involved in fatty acid $\beta$-oxidation, isovaleryl-CoA dehydrogenase and methylbutyryl-CoA dehydrogenase, both involved in branched-chain amino acid metabolism, glutaryl-CoA dehydrogenase, and sarcosine dehydrogenase (1). The patients present with hypoglycemia, metabolic acidosis, hypotonus, hepatomegaly, Reye-like illness, and frequently, neonatal death. Characteristic organic acids derived from these metabolic defects, such as medium-chain dicarboxylic acids, glutarate, ethylmalonate, methylsuccinate, 2-hydroxyglutarate, and isovalerylglycine, are detectable in urine by GC/MS (1). It was also shown by stable isotope dilution analysis that the excretion of hexanoylglycine and suberylglycine could be significantly elevated in urine from patients with GAII, up to an order of magnitude comparable to that observed in patients with medium-chain acyl-CoA dehydrogenase deficiency (2). At the protein level, a primary defect of either ETF, consisting of an $\alpha$-subunit $(\alpha$-ETF) and a $\beta$-subunit ( $\beta$-ETF), or ETF dehydrogenase in GAII was demonstrated (3-7).

Recently, we reported a Japanese boy with GAII due to a defect of $\beta$-ETF biosynthesis revealed by immunoblotting and pulse-chase experiments (7). Subsequently, his parents had another pregnancy and consulted for the prenatal diagnosis of the disease. Prenatal diagnosis of GAII has been reported in several cases $(8-14)$, by either organic acid analysis of the amniotic fluid, or by in vitro oxidation of radioactive substrates by amniocytes. We describe here the prenatal diagnosis of a patient with a mild form of GAII caused by an ETF deficiency accomplished by immunochemical procedures and, retrospectively, by organic acid analysis of amniotic fluid, using a stable isotope dilution method (2). Precise monitoring of the clinical course and the metabolite excretion in early infancy, when the patient had no symptoms, are also described.

\section{CASE PRESENTATION}

The propositus was a Japanese boy, first born of unrelated parents in 1988. He was diagnosed with GAll at the age of 10 mo, based on the results of urinary organic acid analysis by GC/ 
MS (15). A defect in biosynthesis of $\beta$-ETF as the cause of the disease was confirmed by pulse-chase experiments, and the clinical history was reported previously (7).

During a second pregnancy, amniocentesis was carried out at the 17th wk of gestation, and prenatal diagnosis was performed. The pregnancy was continued, even though the immunochemical method described below indicated a strong likelihood that the fetus was affected with GAII due to ETF deficiency.

The baby was a boy, born at 39 wks of gestation, weighing 3912 g. No evident clinical symptoms were noted until early infancy. However, abnormalities in laboratory tests, such as GOT 157 IU/L (normal, <40), LDH 2014 IU/L (normal, <550), CPK $>3000 \mathrm{IU} / \mathrm{L}$ (normal, $<180$ ), were found as early as the 1st day of life, as shown in Table 1. At that time, blood glucose $(4.5$ $\mathrm{mmol} / \mathrm{L}$; normal, 3.9-6.1) and ammonia $(54.0 \mu \mathrm{mol} / \mathrm{L}$; normal, 5-50) were almost normal. The baby was immediately treated with i.v. infusions of $7.5 \%$ glucose. This treatment was continued until $9 \mathrm{~d}$ after birth. Oral supplementation with $10 \mathrm{mg} / \mathrm{d}$ of riboflavin and $30 \mathrm{mg} / \mathrm{kg} / \mathrm{d}$ of $\mathrm{L}$-carnitine were also administered at the 2 nd and the 3 rd d, respectively. As shown in Table 1 , the abnormalities in these laboratory tests were improved at $15 \mathrm{~d}$ of life (GOT 34, LDH 695, and CPK 153). The baby was doing well, and his physical and psychomotor development were within the normal range. He could control his head at 3 mo of age, roll over at $5 \mathrm{mo}$, and sit alone at $6 \mathrm{mo}$.

\section{MATERIALS AND METHODS}

Cell culture. Amniocytes and fibroblasts were cultured in minimum essential medium with $10 \%$ FCS (standard medium).

Immunoblot analysis. Extracts of cultured amniocytes and fibroblasts were applied to SDS/PAGE using $10 \%$ gel, and immunoblot analysis was performed as described previously (16), using the immunoblotting system (Promega Biotec, Madison, WI). Purified rat ETF and the anti-(rat ETF) IgG were prepared as described previously (17).

Pulse-chase experiments. Amniocytes and fibroblasts were grown in 6-cm plastic dishes and pulse-labeled with $200 \mu \mathrm{Ci}$ of $\left[{ }^{35} \mathrm{~S}\right]$ methionine for $1 \mathrm{~h}$ as described (7). Fibroblasts labeled with $\left[{ }^{35} \mathrm{~S}\right]$ methionine were chased with the standard medium for 6 , 24 , and $72 \mathrm{~h}$. Immunoprecipitation and fluorography were done as described (7).

Organic acid analysis. Butyrylglycine, isovalerylglycine, hexanoylglycine, suberylglycine, glutarate, methylsuccinate, and ethylmalonate in amniotic fluid and urine were measured by a stable isotope dilution method previously reported (2), with minor modifications. Uninary organic acids other than the above

Table 1. Time course of serum laboratory tests $(I U / L)$

\begin{tabular}{crrrr}
\hline & GOT & GPT & LDH & \multicolumn{1}{c}{ CPK } \\
\hline $0 \mathrm{~d}$ & 25 & 7 & 737 & 361 \\
$1 \mathrm{~d}$ & 157 & 22 & 2014 & $>3000$ \\
$3 \mathrm{~d}$ & 110 & 35 & 2103 & $>3000$ \\
$5 \mathrm{~d}$ & 76 & 44 & 2178 & 1927 \\
$8 \mathrm{~d}$ & 36 & 30 & 1356 & 318 \\
$12 \mathrm{~d}$ & 43 & 31 & 955 & 209 \\
$15 \mathrm{~d}$ & 34 & 25 & 695 & 153 \\
$24 \mathrm{~d}$ & 25 & 20 & 554 & 169 \\
1 mo 0d & 25 & 21 & 508 & 88 \\
1 mo 15d & 26 & 17 & 490 & 155 \\
2 mo & 46 & 37 & 678 & 168 \\
3 mo & 61 & 55 & 760 & 351 \\
4 mo & 71 & 46 & 1098 & 430 \\
5 mo & 52 & 29 & 1033 & 282 \\
5 mo 19d & 75 & 61 & 1583 & 282 \\
6 mo & 53 & 24 & 1040 & 219 \\
Normal & $<40$ & $<40$ & $<600$ & $<180$ \\
\hline
\end{tabular}

* GPT, glutamic pyruvic transaminase. compounds were analyzed by GC/MS after solvent-extraction, oximation, and trimethylsilylation (standard GC/MS) (15).

\section{RESULTS}

Immunoblot analysis of ETF in amniocytes. As shown in Figure 1, two bands for $\alpha$ - and $\beta$-ETF were detected in both amniocytes and fibroblasts from the controls, but not in the amniocytes from the at-risk fetus.

Pulse-labeling experiment of ETF in amniocytes. In the control fibroblasts and amniocytes, signals for $\alpha$ - and $\beta$-ETF were clearly seen, as shown in Figure 2. In the patient's amniocytes, however, the signal for $\alpha$-ETF but not for $\beta$-ETF was detected. These results indicated the possibility that the fetus was affected with a defect of $\beta$-ETF biosynthesis as in the case of his brother, the propositus.

Immunoblot analysis of ETF in fibroblasts. In the control fibroblasts, two bands for $\alpha$-ETF and $\beta$-ETF were apparently seen (lanes 2 and 4 in Figure 3), but no bands for the two subunits were detected in fibroblasts from the baby (lane 3), results similar to those in the amniocytes.

Pulse-chase experiments of ETF in fibroblasts. The results of the pulse-chase experiment of ETF using the fibroblasts are shown in Figure 4. In the control, signals for both $\alpha$-ETF and $\beta$ ETF were clearly seen after a 1-h pulse, a 6-h chase, a 24-h chase, and a 72-h chase (lanes $1-4$ ). In the patient's fibroblasts (lanes $5-8$ ), no signal for $\beta$-ETF was detected in any lane, whereas the signal for $\alpha$-ETF was seen faintly after a 1 -h pulse and a 6 -h chase, and disappeared after a 24 -h chase. These results confirmed that this patient was also affected with GAII caused by a defect of $\beta$-ETF biosynthesis.

Organic acids in amniotic fluid. Table 2 summarizes the quantitative results of three acylglycines and three organic acids determined by stable isotope-dilution analysis (2). Only the glutarate concentration in the at-risk amniotic fluid was evidently

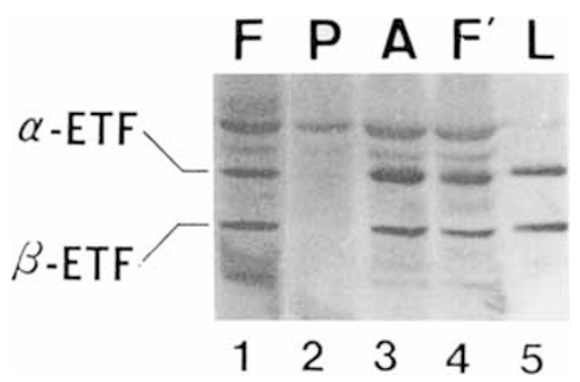

Fig. 1. Prenatal diagnosis of GAII by immunoblot analysis of ETF in cultured amniocytes. Lanes $l$ and 4, control fibroblasts; lane 2, patient's amniocytes; lane 3, control amniocytes; and lane 5, control human liver at autopsy. The amount applied to each lane corresponded to $50 \mu \mathrm{g}$ of protein in extracts from amniocytes or fibroblasts and $5 \mu \mathrm{g}$ of liver extract, respectively. $F$ and $F^{\prime}$, control fibroblasts; $P$, patient's amniocytes; $A$, control amniocytes; and $L$, control human liver at autopsy.

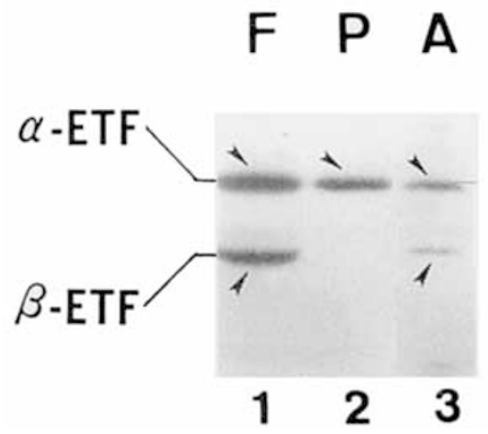

Fig. 2. Prenatal diagnosis of GAIl by pulse labeling of ETF in cultured amniocytes. Lane 1, control fibroblasts; lane 2, patient's amniocytes; and lane 3, control amniocytes. Abbreviations are the same as in Figure 1. 


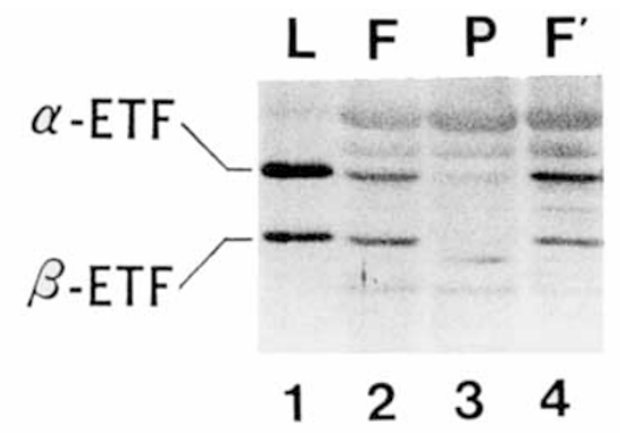

Fig. 3. Immunoblots of ETF in fibroblasts obtained after birth. Lane 1 , control human liver; lanes 2 and 4, control fibroblasts; and lane 3, the patient's fibroblasts. The amount applied was the same as in Figure 1. Abbreviations are the same as in Figure 1.

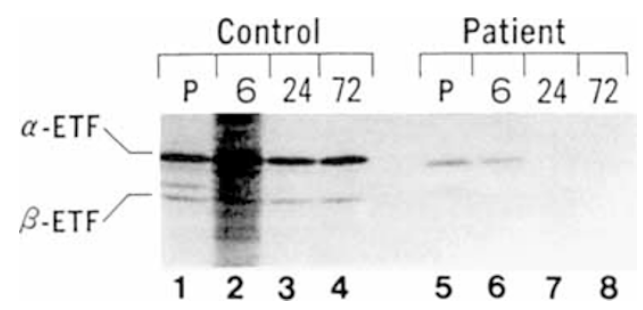

Fig. 4. Pulse-chase experiments of ETF in the patient's fibroblasts obtained after birth. Lanes $1-4$, control fibroblasts; lanes 5-8, patient's fibroblasts. $P, 1-\mathrm{h}$ pulse; 6,24 , and 72 , chase for $6 \mathrm{~h}, 24 \mathrm{~h}$, and $72 \mathrm{~h}$, respectively.

increased about 5-10 times that of the controls. A mild increase of ethylmalonate, methylsuccinate, butyrylglycine, isovalerylglycine, or hexanoylglycine was also noted, although the diagnostic significance of such an increase was not clear.

Urinary excretion of organic acids after birth. Table 3 summarizes the time course of metabolite excretion in early infancy. By use of a standard GC/MS method, no significant abnormalities in excretion of adipate, suberate, sebacate, glutarate, ethylmalonate, and 2-hydroxyglutarate were noted at $9 \mathrm{~h}$ after birth. However, an increased excretion of hexanoylglycine was detected by stable isotope dilution analysis in the same specimen. 2 Hydroxyglutarate and ethylmalonate excretion exceeded the respective range of control values within $24 \mathrm{~h}$, a pathologic excretion of the remaining organic acids and acylglycines occurred within a week. An increase in ethylmalonate excretion was found after $24 \mathrm{~h}$; methylsuccinate, glutarate, and butyrylglycine, at $5 \mathrm{~d}$; and isovalerylglycine, at $8 \mathrm{~d}$. Phenylpropionylglycine was within a normal range throughout the entire period of investigation. The findings of the urinary organic acids in his older brother, the proband, at 14 mo and $2 \mathrm{y}$ of age, were also shown in Table 3 for comparison. The abnormalities in the urinary organic acids of the baby were qualitatively and quantitatively similar to those previously observed in his brother, the propositus, about $1-3 \mathrm{wk}$ after birth.

\section{DISCUSSION}

In this study, the usefulness of immunochemical methods in the prenatal diagnosis of GAII and the usefulness of precise follow-up of metabolite excretion immediately after birth in a patient with a mild form of GAII were demonstrated for the first time.

Clinical heterogeneity of GAII has been reported, and GAII has been classified into the three following forms (1): neonatal onset form with congenital anomalies; neonatal onset form without anomalies; and late onset, mild form. Most patients with the neonatal onset forms die within the first week of life. Patients with the late onset form may survive longer, having episodic attacks such as hypotonia, hypoglycemia, metabolic acidosis, or sometimes Reye's syndrome-like illness. The propositus in this report could be described as having the mild form rather than the neonatal form, although he was hospitalized with neonatal transient respiratory distress. He has survived longer, satisfactorily recovering from intercurrent infections or diarrhea, and could walk alone at the age of 21 mo.

The baby in this study is likely to belong to the group with the late onset, mild form of GAII. Up to date, his weight gain and development have been within normal ranges and he had no evident symptoms in infancy while receiving oral supplementation with L-carnitine and riboflavin. However, it should be underscored that a transient elevation in serum GOT, LDH, and CPK was noted in the early neonatal period and persisted thereafter. Had he not received such care since early infancy, we believe that he might have presented with symptoms as serious as his brother, who suffered gross motor delay and recurrent acute episodes of illness. These observations suggest that patients with a mild form of GAII could present in the immediate neonatal period, and for variable periods of time thereafter, with only minor and difficult to detect biochemical abnormalities. These results also indicate that early and aggressive treatment could play a role in the long-term outcome of these patients.

In the neonatal, severe form of GAII, a characteristic pattern of organic acids is frequently detectable from the early neonatal period $(1,18)$. In contrast, in the mild form, the diagnosis may be more difficult because the organic aciduria is considerably less pronounced and often intermittent $(1,19-21)$. In this case, an increased excretion of hexanoylglycine in urine was detected by stable isotope dilution analysis immediately after birth and continued thereafter. Other organic acids characteristic of GAII gradually increased to values similar to those observed in his sibling, the propositus, at $1-3 \mathrm{wk}$ after birth. As in the case of other fatty acid oxidation disorders like medium-chain acyl-CoA dehydrogenase deficiency (2), a warning should be made that these abnormalities could be easily missed by a standard GC/ MS method. Although several organic acids indicative of GAII might be present in urine, to some degree, weeks or months after

Table 2. Concentration of acylglycines and organic acids in amniotic fluid $(\mu \mathrm{mol} / L)^{*}$

\begin{tabular}{lcccccc}
\hline \multicolumn{1}{c}{ Amniotic fluid } & BG & IVG & HG & EMA & MSA & GLU \\
\hline $\begin{array}{l}\text { Pregnancy at risk for GAII } \\
\text { Japanese controls } \dagger\end{array}$ & 1.19 & 0.43 & 1.61 & 1.33 & 0.56 & 5.91 \\
$\quad$ Control 1 & 0.49 & 0.23 & 0.41 & 0.47 & 0.33 & 0.50 \\
$\quad$ Control 2 & 0.44 & 0.15 & 0.48 & 0.41 & 0.23 & 0.78 \\
$\begin{array}{l}\text { Caucasian controls } \\
\quad(n=12) \ddagger\end{array}$ & & & & & & \\
$\quad$ Mean \pm SD & $0.12 \pm 0.02$ & $0.18 \pm 0.04$ & $0.23 \pm 0.03$ & $0.69 \pm 0.11$ & $0.09 \pm 0.05$ & $0.72 \pm 0.26$ \\
$\quad$ Range) & $(0.08-0.15)$ & $(0.11-0.23)$ & $(0.19-0.28)$ & $(0.55-0.91)$ & $(0.04-0.20)$ & $(0.44-1.33)$ \\
\hline
\end{tabular}

* BG, n-butyrylglycine; IVG, isovalerylglycine; HG, n-hexanoylglycine; EMA, 2-ethylmalonic acid; MSA, 2-methylsuccinic acid; and GLU, glutaric acid.

+ Amniocentesis of controls 1 and 2 performed in the 18th gestational week because of prenatal diagnosis of the other inherited diseases.

$\ddagger$ Amniocentesis in the 16 th-19th gestational week because of advanced maternal age. 
Table 3. Time course of urinary metabolite excretion after birth (mmol/mol creatinine**

\begin{tabular}{|c|c|c|c|c|c|c|c|c|c|c|c|c|}
\hline \multirow[b]{2}{*}{ Time after birth } & \multicolumn{8}{|c|}{$\mathrm{GC} / \mathrm{MS}$ stable isotope dilution analysis } & \multicolumn{4}{|c|}{ Standard GC/MS analysis } \\
\hline & BG & IVG & $\mathrm{HG}$ & PPG & $\mathrm{SG}$ & EMA & MSA & GLU & $2 \mathrm{HG}$ & ADI & SUB & SEB \\
\hline \multicolumn{13}{|l|}{ Subject } \\
\hline $9 \mathrm{~h}$ & 0.13 & 5.18 & 2.98 & 0.06 & 4.22 & 11.08 & 2.53 & 3.17 & 15.02 & 2.01 & 1.49 & 1.62 \\
\hline $10 \mathrm{~h}$ & 0.62 & 2.59 & $\overline{3.55}$ & 0.09 & 6.00 & 14.97 & 3.72 & 5.14 & 10.27 & 4.88 & 3.90 & 2.29 \\
\hline $24 \mathrm{~h}$ & 0.26 & 1.40 & 1.48 & 0.13 & $\overline{3.24}$ & 19.60 & 3.35 & 4.55 & 32.42 & 7.20 & 4.74 & 2.85 \\
\hline $31 \mathrm{~h}$ & 0.40 & 1.19 & 2.01 & 0.05 & 3.72 & $\overline{19.30}$ & 6.17 & 6.53 & 11.43 & 8.28 & 8.15 & 2.46 \\
\hline $48 \mathrm{~h}$ & 0.30 & 2.97 & 3.36 & 0.19 & 21.92 & 37.16 & 9.16 & 8.11 & 44.75 & 14.86 & 13.12 & 1.23 \\
\hline $3 d$ & 0.15 & 2.70 & 10.71 & 0.18 & 13.37 & 41.18 & 10.61 & 7.92 & 22.60 & 23.22 & 25.00 & 15.16 \\
\hline $5 \mathrm{~d}$ & 3.03 & 5.47 & 3.33 & 0.01 & 32.04 & 34.05 & 17.68 & 29.32 & 54.32 & $\frac{2.25}{16.33}$ & $\frac{2.00}{9.22}$ & 0.28 \\
\hline $7 \mathrm{~d}$ & 0.94 & 8.70 & 2.19 & 0.07 & 1.28 & 39.04 & 6.17 & 11.05 & 73.39 & 16.33 & 13.12 & 4.08 \\
\hline $11 \mathrm{~d}$ & 2.20 & 38.36 & 6.69 & 0.01 & 3.96 & 68.74 & 11.92 & 29.17 & $\overline{78.59}$ & 24.07 & 30.91 & 6.04 \\
\hline $14 \mathrm{~d}$ & 0.34 & $\overline{36.49}$ & 5.85 & 0.01 & 10.54 & 41.19 & 7.84 & 29.74 & 66.00 & 35.83 & 40.13 & 16.22 \\
\hline $23 \mathrm{~d}$ & 1.47 & 97.24 & 9.16 & 0.12 & 68.61 & 118.73 & 25.01 & 88.99 & 334.89 & 42.96 & 25.85 & 20.59 \\
\hline $30 \mathrm{~d}$ & 0.85 & 49.58 & 1.74 & 0.01 & 5.19 & 47.20 & 5.76 & 8.65 & 149.08 & 53.02 & 20.85 & 3.41 \\
\hline $45 \mathrm{~d}$ & 0.54 & 138.22 & 4.87 & 0.01 & 117.35 & 47.32 & 7.69 & 21.26 & 334.70 & 61.84 & 40.00 & 2.18 \\
\hline $60 \mathrm{~d}$ & 1.12 & 55.95 & 1.87 & 0.47 & 82.59 & 51.01 & 8.30 & 10.19 & 22.70 & 45.82 & 15.33 & 2.46 \\
\hline $2 \mathrm{mo} 17 \mathrm{~d}$ & 2.53 & 247.54 & 7.48 & 0.63 & 2.85 & 35.11 & 3.82 & 7.17 & 155.05 & 28.95 & 18.90 & 7.22 \\
\hline $3 \operatorname{mo} 7 d$ & 1.18 & 71.60 & 26.97 & 0.48 & 15.37 & 51.66 & 6.75 & 7.22 & 166.48 & 128.87 & 68.84 & 5.48 \\
\hline $4 \mathrm{mo} 0 \mathrm{~d}$ & 0.98 & 165.33 & 15.73 & 0.43 & 9.35 & 50.52 & 5.48 & 5.03 & 173.67 & 31.19 & 14.42 & 0.67 \\
\hline $4 \mathrm{mo} 14 \mathrm{~d}$ & 1.16 & 73.49 & 13.06 & 0.57 & 11.02 & 62.26 & 7.79 & 7.12 & 142.66 & 93.96 & 50.72 & 3.97 \\
\hline $5 \mathrm{mo} 0 \mathrm{~d}$ & 0.24 & 28.48 & 19.49 & 0.44 & 14.05 & 28.61 & 2.93 & 2.72 & 139.58 & 292.64 & 126.57 & 123.96 \\
\hline $5 \mathrm{mo} 12 \mathrm{~d}$ & 2.95 & 32.61 & 34.49 & 0.81 & 14.94 & 83.99 & 9.93 & 3.71 & 214.83 & 87.38 & 26.76 & 12.03 \\
\hline \multicolumn{13}{|l|}{$\begin{array}{l}\text { Sibling (proposi- } \\
\text { tus) }\end{array}$} \\
\hline 1 у 2 mo & 0.20 & 58.27 & 4.52 & 0.04 & 12.87 & 38.81 & 9.72 & 6.96 & 143.18 & 75.00 & 23.96 & 3.92 \\
\hline 2 y $0 \mathrm{mo}$ & 2.18 & 319.37 & 64.91 & 0.03 & 28.10 & 83.37 & 1.17 & 4.23 & 841.79 & 91.64 & 33.77 & 3.52 \\
\hline \multicolumn{13}{|l|}{ Controls } \\
\hline $\begin{array}{l}\text { Mean } \\
\text { (Range) }\end{array}$ & $\begin{array}{c}0.65 \dagger \\
(0.02-1.64)\end{array}$ & $\begin{array}{c}3.48 \dagger \\
(0.21-10.2)\end{array}$ & $\begin{array}{c}0.57 \ddagger \\
(0.13-1.24)\end{array}$ & $\begin{array}{c}0.29 \ddagger \\
(<0.01-0.72)\end{array}$ & $\begin{array}{c}1.98 \ddagger \\
(<0.01-5.38)\end{array}$ & $\begin{array}{c}6.94 \dagger \\
(0.43-17.3)\end{array}$ & $\begin{array}{c}4.27 \dagger \\
(0.34-11.8)\end{array}$ & $\begin{array}{c}4.94 \dagger \\
(0.51-13.0)\end{array}$ & $\begin{array}{c}32.42 \S \\
(0.00-72.2)\end{array}$ & $\begin{array}{c}13.78 \S \\
(2.32-22.1)\end{array}$ & $\begin{array}{c}6.69 \S \\
(0.71-15.9)\end{array}$ & $\begin{array}{c}3.97 \S \\
(0.00-9.85)\end{array}$ \\
\hline
\end{tabular}

* BG, IVG, HG, EMA, MSA, and GLU have the same meanings as in Table 2; PPG, phenylpropionyl-glycine; SG, suberylglycine; 2HG, 2-hydroxyglutarate; ADI, adipate; SUB, suberate; and SEB, sebacate. Underlined values indicate the first occurrence of abnormal excretion for each metabolite.

$+n=25$, age $7 \mathrm{~d}-9$ mo, same urine samples used as normal controls in Reference 2

$\$$ From Reference 2 .

$\S n=20,1 \mathrm{mo}-10 \mathrm{mo}$. 
birth, our results confirm the findings by Loehr et al. (6) that patients with a phenotypically mild form of GAII might not be detected by standard GC/MS analysis.

The application of organic acid analysis of amniotic fluid by GC/MS or in vitro oxidation of radioactive substrates in amniocytes have been reported as prenatal diagnosis of GAII (8-14). In vitro oxidation assay, however, is not always an available option for prenatal diagnosis because it requires a large number of cells and is time-consuming. This study represents the first report of prenatal diagnosis of a fetus with GAII using immunochemical methods such as immunoblotting and pulse labeling. These procedures require a relatively small number of cells and yield reliable results. However, their only limitation is the necessity to pinpoint at the protein product level the site of the defect (ETF or ETF dehydrogenase) in the propositus.

Prenatal diagnosis by amniotic fluid analysis using a stable isotope dilution method have been reported in several organic acidurias such as methylmalonic acidemia (22-24), isovaleric acidemia (25), and glutaric aciduria type I (26). In many of these cases, a substantial difference in concentration of the diagnostic compounds between normal controls and the affected fetus has been detectable. The organic acid analysis of amniotic fluid using such a method (2) was also used for the prenatal diagnosis of our case. Prenatal diagnosis of GAII by the organic acid analysis of amniotic fluid has been reported in several cases in which the propositus was likely to belong to the neonatal onset form of GAII (8-14). The levels of glutarate and 2-hydroxyglutarate in the amniotic fluid from the affected fetus were about 20 and 3 times those in the normal controls, respectively, in the report by Boue et al. (10); the levels of glutarate, adipate, sebacate, and suberate were about $15,10,7$, and 3 times higher than normal, respectively, as reported by Jakobs et al. (11); the level of glutarate was 10 times normal as reported by Henderson et al. (14). In these reports, an increased concentration of glutarate in the amniotic fluid was noted as one of the most reliable indices for the prenatal diagnosis of GAII, although the amount may be much smaller than that detectable in amniotic fluid from fetuses affected with glutaric aciduria type I (26). Consistently, glutarate concentration in the amniotic fluid was increased about 5 times greater than reference values. Other metabolites such as ethylmalonate, methylsuccinate, butyrylglycine, and hexanoylglycine were also elevated in the amniotic fluid. Although these findings were indeed consistent with the results obtained by immunoblotting and pulse labeling experiments and the elevated glutarate concentration, the reproducibility of these results should be further evaluated before the attribution of any diagnostic significance. In addition, it is interesting that the urinary excretion of glutarate as well as the other metabolites was almost normal immediately after birth, although glutarate concentration was increased in the amniotic fluid at the $17 \mathrm{th}$ wk of gestation.

Rapid, reliable, and complementary procedures are essential for the prenatal diagnosis of inherited diseases. Under this perspective, the immunochemical methods used in this study for the prenatal diagnosis of GAII provided reliable results, requiring a smaller number of cultured cells. Organic acid determination in amniotic fluid by stable isotope dilution analysis also provided significant diagnostic information, although the gap in concentration between normal and affected cases was relatively small. The tandem application of these procedures is likely to provide a rapid and more reliable prenatal diagnosis of ETF deficiency.

Acknowledgments. The authors thank Prof K. Tanaka, Yale University, Department of Genetics, for his assistance with our study and Prof Y. Wada and Dr. M. Kobayashi, Nagoya City University, Department of Pediatrics, for providing the amniotic fluid.

\section{REFERENCES}

1. Frerman FE, Goodman SI 1989 Glutaric acidemia type II and defects of the mitochondrial respiratory chain. In: Scriver CR, Beaudet AL, Sly WS, Valle $\mathrm{D}$ (eds) The Metabolic Basis of Inherited Metabolic Disease. McGraw-Hill, New York, pp 915-931

2. Rinaldo P, O'Shea JJ, Coates PM, Daniel HE, Stanley CA, Tanaka K 1988 Medium-chain acyl-CoA dehydrogenase deficiency diagnosis by stable-isotope dilution measurement of urinary $n$-hexanoylglycine and 3-phenylpropionylglycine. N Engl J Med 319:1308-1313

3. Frerman FE, Goodman SI 1985 Deficiency of electron transfer flavoprotein ubiquinone oxidoreductase in glutaric aciduria type II fibroblasts. Proc Natl Acad Sci USA 82:4517-4520

4. Ikeda Y, Keese SM, Tanaka K 1986 Biosynthesis of electron transfer flavoprotein in a cell-free system and in cultured human fibroblasts. Defect in the alpha subunit synthesis is a primary lesion in glutaric aciduria type II. $\mathrm{J}$ Clin Invest 78:997-1002

5. Rhead WJ, Wolff JA, Lipson M, Falace P, Desai N, Fritchman K, Moon A Sweetman L 1987 Clinical and biochemical variation and family studies in the multiple acyl-CoA dehydrogenation disorders. Pediatr Res 21:371-376

6. Loehr JP, Goodman SI, Frerman FE 1990 Glutaric aciduria type II: heterogeneity of clinical and biochemical phenotypes. Pediatr Res 27:31I-315

7. Yamaguchi S, Orii T, Suzuki Y, Maeda K, Oshima M, Hashimoto T 199 Newly identified forms of electron transfer flavoprotein deficiency in two patients with glutaric aciduria type II. Pediatr Res 29:60-63

8. Mitchel G, Saudubray JM, Benoit Y, Rocchiccioli F, Charpentier C, Ogier H Boue J 1983 Antenatal diagnosis of glutaric aciduria type II. Lancet 1:1099

9. Bennett MJ, Curnock DA, Engel PC, Shaw L, Gray RGF, Hull D, Patrick AD Pollitt RJ 1984 Glutaric aciduria type II: biochemical investigation and treatment of a child diagnosed prenatally. J Inherited Metab Dis 7:57-61

10. Boue J, Charmars RA. Tracey BM, Watson D, Gray RGF, Keeling JW, King GS, Pettit BR, Lindenbaum RH, Rocchiccioli F, Saudubray JM 1984 Prenatal diagnosis of dysmorphic neonatal lethal type II glutaric aciduria. Lancet 1:846-847

11. Jakobs C, Sweetman L, Wadman SK, Duran M, Saudubray JM, Nyhan WL 1984 Prenatal diagnosis of glutaric aciduria type II by direct chemical analysis of dicarboxylic acids in amniotic fluid. Eur J Pediatr 141:153-157

12. Sweetman L 1984 Prenatal diagnosis of the organic acidurias. J Inherited Metab Dis 7(suppl 1): 18-22

13. Chalmers RA, Tracey BM, King GS, Pettit B, Rocchiccioli F, Saudubray JM Gray RGF, Boue J, Keeting JW, Lindenbaum RH 1985 The prenatal diagnosis of glutaric aciduria type II, using quantitative GC-MS. J Inherited Metab Dis 8(suppl 2):145-146

14. Henderson HE, Balla R, De Jong G, Peik CJ, Mienie LJ, Erasmus E 1987 Postnatal and antenatal laboratory diagnosis of glutaric aciduria II in a South African family. S Afr Med J 71:589-591

15. Yamaguchi S, Orii T, Yasuda K, Kohno Y 1987 A case of glutaric aciduria type I with unique abnormalities in the cerebral CT findings. Tohoku $J$ Exp Med 151:293-299

16. Yamaguchi S, Orii T, Sakura N, Miyazawa S, Hashimoto T 1988 Defect in biosynthesis of mitochondrial acetoacetyl-CoA thiolase in cultured fibroblasts from a boy with 3-ketothiolase deficiency. J Clin Invest 81:813-817

17. Furuta S, Miyazawa S, Hashimoto T 1981 Purification and properties of rat liver acyl-CoA dehydrogenases and electron transfer flavoprotein. J Biochem 90:1739-1750

18. Przyrembel $\mathrm{H}$, Wendel $\mathrm{U}$, Becker $\mathrm{K}$, Bremer $\mathrm{HJ}$, Bruinvis $\mathrm{L}$, Ketting $\mathrm{D}$ Wadman SK 1976 Glutaric aciduria type II: report on a previously undescribed metabolic disorder. Clin Chim Acta 66:227-239

19. Mantagos S, Genel M, Tanaka K 1979 Ethylmalonic-adipic aciduria in vivo studies indicating deficiency of activities of multiple acy-CoA dehydrogenases. J Clin Invest 64:1580-1589

20. Rinaldo P, Welch RD, Previs SF, Schmidt-Sommerfeld E, Gargus JJ, O'Shea JJ, Zinn AB 1991 Ethylmalonic/adipic aciduria: effects of oral mediumchain triglycerides, carnitine, and glycine on urinary excretion of organic acids, acylcarnitines, and acylglycines. Pediatr Res 30:216-221

21. Shimizu N, Yamaguchi S, Orii T, Previs SF, Rinaldo P 1991 Mass spectrometric analysis of metabolite excretion in five Japanese patients with the late onset form of glutaric aciduria type II. Biol Environ Mass Spectrom 20:479483

22. Naylor G, Sweetman L, Nyhan WL, Hornbeck C, Griffiths J, Merche, Brandange $S 1980$ Isotope dilution analysis of methylcitric acid in amniotic fluid for the prenatal diagnosis of propionic and methylmalonic acidemia. Clin Chim Acta 107:175-183

23. Tretz FK, Schmidt H, Tauscher B, Depene E, Baumgartner R, Hammersen $G$, Kochen W 1981 Improved prenatal diagnosis of methylmalonic acidemia: mass fragmentography of methylmalonic acid in amniotic fluid and maternal urine. Eur J Pediatr 137:216-266

24. Zinn AB, Hine DG, Mahoney M, Tanaka K 1982 The stable isotope dilution method for measurement of methylmalonic acid: a highly accurate approach to the prenatal diagnosis of methylmalonic acidemia. Pediatr Res 16:740745

25. Hine DG, Hack AM, Goodman SI, Tanaka K 1986 Stable isotope dilution analysis of isovalerylglycine in amniotic fluid and urine and its application for the prenatal diagnosis. Pediatr Res 20:222-226

26. Goodman SI, Gallegos DA. Pullin CJ, Halpern B, Truscott RJW, Wise G Wilcken B, Ruan ED, Whelan DT 1980 Antenatal diagnosis of glutaric aciduria. Am J Hum Genet 32:695-699 